\title{
DALLA PARTE DELLE BAMBINE Analisi del contributo di Bianca Pitzorno alla letteratura per l'infanzia
}

\author{
Elena Tosi \\ Università degli studi di Modena e Reggio Emilia \\ Facoltà di Scienze della Formazione Primaria \\ Dipartimento di Educazione e Scienze Umane
}

\section{Riassunto}

Il saggio offre una panoramica sul contributo dato dalla scrittrice italiana Bianca Pitzorno alla letteratura per l'infanzia. Questa autrice è importante in ambito nazionale e internazionale, poiché ha dedicato tutta la vita a scrivere storie di bambine e adolescenti femmine, laddove la letteratura le relegava in un angolo, affidando loro ruoli secondari o caratteri deboli e sottomessi. Nella letteratura troviamo altri autori che hanno scritto di bambine (come Carroll, Michaelis, Lindgren, Dahl e Petrosino), ma mai nessuno vi si è dedicato totalmente come la Pitzorno. Confrontando quindi alcuni protagonisti maschili dei Classici (in particolare quelli di Calvino, Collodi, De Amicis, Kipling e Malot) con quelli femminili della Pitzorno, risulta che queste non hanno nulla da invidiare ai "colleghi" maschi.

Parole chiave: Pitzorno, bambine, letteratura, stereotipi, genere 


\section{Introduzione: Bianca Pitzorno come Tusitala}

Bianca Pitzorno ${ }^{[1]}$ può essere considerata un'eccezione nel panorama della letteratura per l'infanzia, italiana e internazionale. Questo è dovuto al fatto che lei abbia dedicato l'intera sua carriera di scrittrice a scrivere romanzi che avessero come protagoniste bambine e adolescenti femmine, schierandosi apertamente dalla loro parte e, per citare la scrittrice stessa, proponendosi di narrare l'epos del suo popolo. ${ }^{[2]}$ Fernando Rotondo definisce addirittura la Pitzorno la "signora della letteratura per l'infanzia". ${ }^{[3]}$

Nel suo volume Storia delle mie storie $e^{[4]}$ distingue due categorie di autori per bambini: quella degli "educatori" e quella dei "narratori puri". Nella prima categoria incappano autori che di solito "piacciono molto agli adulti che comprano i libri, e poco ai piccoli che devono leggerli" ${ }^{[5]} \mathrm{e}$ che non riescono a resistere alla tentazione di inserire una predica morale all'interno delle storie. Nella seconda, invece, si annoverano i Tusitala, termine che nella lingua delle isole Samoa significa "colui che racconta". La Pitzorno fa rientrare se stessa in questa categoria:

Noi Tusitala invece raccontiamo con l'unica preoccupazione dello charme, dell'incanto della storia e della parola; cerchiamo non di proporre modelli ideali, ma di "imitare il vero" dell'esperienza, delle passioni e dei desideri dei bambini. Sappiamo che i piccoli non sono semplici né innocenti, come vorrebbero gli adulti smemorati. Sappiamo che nutrono forti passioni, che sono scossi da emozioni profonde. ${ }^{[6]}$

\footnotetext{
[1] Bianca Pitzorno nasce a Sassari nel 1942. Vive la sua adolescenza a Sassari, in Sardegna, e questa terra farà da sfondo a molti dei suoi romanzi. Da molti dei suoi ricordi risalenti a questo periodo, che sono rimasti indelebili nella sua mente, verrà poi attinto il materiale per diversi dei suoi romanzi. La maggior parte di questi sono raccolti nel volume Storia delle mie storie (Nuove Pratiche Editrice, Parma, 1995), dove racconta di prima mano le sue esperienze, le sue letture, i motivi che le danno la possibilità di identificarsi così facilmente con bambine e adolescenti. Successivamente, intraprendendo gli studi universitari, si trasferisce a Cagliari, dove frequenta la facoltà di Lettere e si specializza in archeologia preistorica. Il periodo successivo, che trascorre a Milano, la segna profondamente. Lavora per diversi anni presso la RAI, per la quale si occupa di programmi culturali - anche per bambini. Durante questi anni impara molto ma si rende conto di non essere fatta per il lavoro déquipe, quindi decide di dedicarsi completamente ad unattività che aveva accantonato, convinta dagli amici che litterae non dant panem: quella di scrittrice. Da quel momento pubblicherà più di quaranta libri, di cui quasi la totalità con protagoniste bambine e adolescenti femmine.

[2] PITZORNO, B., 1995, Storia delle mie storie, Nuove Pratiche Editrice, Parma, p. 34.

[3] ROTONDO, F., in CATARSI, E., (a cura di), 2002, Gianni Rodari e la letteratura per l'infanzia, Edizioni Del Cerro, Pisa, p. 89

[4] PITZORNO, B., 1995, Storia delle mie storie, Nuove Pratiche Editrice, Parma.

[5] Ivi, p. 23

[6] Ivi, p. 35
} 
Bianca Pitzorno quindi non si è dimenticata di essere stata una bambina, e soprattutto cosa significhi essere una bambina. Per questo motivo ha deciso, nel corso del tempo, di scrivere romanzi che hanno quasi esclusivamente per protagoniste bambine o ragazzine. Questo perché le conosce profondamente, perché ci è passata. Ha frequentato classi femminili, ha vissuto molti anni in ambienti femminili e lei stessa è stata una bambina molto consapevole di esserlo.

Fonda la collana Gaia alla Mondadori per dare nuova dignità alle "bambine di carta", fino a quel momento remissive e servizievoli. Fino ad allora pochi sono gli esempi significativi, almeno per l'Italia, di tentativi di schierarsi "dalla parte delle bambine". ${ }^{[7]}$

La Pitzorno quindi attinge a piene mani dalla sua vita, dalla sua esperienza e da quella delle bambine che si confidano con lei, per dare la possibilità a quella "bambina molto arrabbiata" ${ }^{[8]}$ di esprimersi e di dare voce ad altrettante bambine, arrabbiate almeno quanto lei.

I suoi personaggi e storie sono simbolo di una nuova consapevolezza da parte dell'editoria e delle lettrici, che leggono mediamente più dei coetanei maschi e non si accontentano più dei cosiddetti "libri rosa". Nella letteratura per l'infanzia, infatti, solitamente è rara la presenza di personaggi femminili che siano forti, coraggiosi, indipendenti, intraprendenti: queste caratteristiche sono riservate a quelli maschili.

Tuttavia sarebbe scorretto e riduttivo sostenere che nella storia della letteratura per l'infanzia non esistano altri personaggi femminili altrettanto forti e significativi, almeno quanto lo siano ad esempio i personaggi pitzorniani di Lavinia, Prisca o Barbara. Questi condividono lo spirito di avventura, l'autonomia, il fatto di non rientrare - per motivi differenti negli schemi che la società ha previsto per loro. La letteratura per l'infanzia grazie a questi personaggi cambia dal punto di vista dei contenuti, perché finalmente "i bambini e i ragazzi vengono considerati protagonisti e osservatori perspicaci della vita, nonché soggetti umanamente complessi”. ${ }^{[9]}$

[7] Tra gli altri, ricordiamo il saggio della scrittrice Elena Gianini Belotti, che nel 1973 pubblica per Feltrinelli un saggio che poi diventerà una pietra miliare per la lotta agli stereotipi di genere, Dalla parte delle bambine: in esso si sostiene che la formazione di maschi e femmine derivi in gran parte da input imposti dalla società piuttosto che da inclinazioni personali.

[8] Cfr. PITZORNO, B., 1995, Storia delle mie storie, Nuove Pratiche Editrice, Parma, p. 36.

[9] Cfr. BLEZZA PICHERLE, S., 2004, Libri, bambini, ragazzi - Incontri tra educazione e letteratura, Vita e Pensiero, Milano, p. 137. 


\section{Le bambine della letteratura per l'infanzia}

Chi fa da apripista è sicuramente Alice di Alice nel paese delle meraviglie (1865) di Lewis Carroll (1832-1898), che è tutto fuorché la tipica bambina di età vittoriana: non è dolce né passiva, è anzi determinata e non ha nessuna intenzione di sottostare alle prepotenze di chi si trova attorno a lei. Alice è circondata da adulti che non la convincono, che dispensano massime che per primi non seguono. Pone spesso domande scomode, a cui gli adulti non sono disposti a rispondere. Non è quindi un caso che Alice sia un personaggio così amato dai bambini (ma anche da molti adulti): risulta essere sempre critica rispetto al mondo che la circonda e, se si trova di fronte ad un'ingiustizia, protesta ardentemente, richiedendo spiegazioni.

L'esempio di Carroll è seguito da tre autori nordici: Karin Michaelis (1872-1950), Astrid Lindgren (1907-2002) e Roald Dahl (1916-1990). Al pari di Alice, infatti, anche Bibi di Bibi bambina del nord (Karin Michaelis, 1929) è molto irriverente. Inoltre è indipendente e ribelle: si rifiuta di andare a scuola, preferendo invece viaggiare da sola per la Danimarca. Durante queste avventure in solitaria scrive numerose lettere al padre, facendogli un resoconto dettagliato rispetto alle persone incontrate ed ai luoghi visitati. Silvia Blezza Picherle ritrae questa bambina in modo molto efficace:

Se volessimo tracciare in breve il suo ritratto, potremmo dire che è allegra ed entusiasta della vita, desiderosa di conoscere, piena di iniziativa e di intraprendenza, coraggiosa nell'affrontare situazioni difficili e talvolta pericolose, tanto volitiva e caparbia da portare a termine qualsiasi impresa o azione decida di compiere. Siamo di fronte ad una personalità che lotta con grande forza e coraggio contro tutti coloro che cercano di ostacolare le sue decisioni e le sue scelte. Di lei colpisce il comportamento talmente libero e spontaneo da apparire trasgressivo rispetto ai canoni educativi dell'epoca, per cui il suo entusiasmo e la sua esuberanza fanno sì che ella assuma molti atteggiamenti ritenuti sconvenienti. ${ }^{[10]}$

Per questi modi di fare, che non si addicono ad una bambina perbene, Bibi durante il periodo di supremazia nazista in Germania viene [10] Ivi, p. 104. 
messa al bando insieme a molti altri libri per l’infanzia. Bibi è indipendente, libera da ogni schema: è considerata una bambina sovversiva e per questo motivo non può essere tollerata dal Regime tedesco. Tuttavia non viene censurata dal Fascismo italiano, probabilmente perché la sua ribellione viene sottovalutata. In un articolo per la rivista "Liber" Donatella Ziliotto analizza le ragioni per cui Bibi ha segnato così tanto la generazione delle bambine degli anni Quaranta e scrive:

Negli anni '40 fu Bibi - La bambina venuta dal nord della danese Karin Michaelis a segnare le scelte della generazione di bambine di quegli anni, mortificate dalle restrizioni morali e materiali di quel periodo, immobilizzate dagli allarmi, dai coprifuochi, dalla fame, mentre Bibi se ne andava in bicicletta per il suo paese, incontrando vagabondi, adottando cicogne, infischiandosene dei nobili e dei ricchi. Se in Germania tutte le copie del libro vennero bruciate, i nazisti non sbagliarono: dietro i romanzi della Michaelis c'era una donna che nella sua isola aveva nascosto Bertolt Brecht, Albert Einstein, Walter Benjamin, Oskar Kokoschka. Solo l'ottusità dei fascisti fece sì che non s'accorgessero che attraverso i libri di Bibi le bambine di allora avevano scoperto la democrazia. ${ }^{[1]}$

Come Bibi, anche Pippi di Astrid Lindgren (Pippi Calzelunghe, 1945) rivendica la sua indipendenza. Pippi è una bambina anticonvenzionale in tutto. Senza madre, durante i primi anni della sua vita viaggia in nave con il padre marinaio e vede il mondo, con i suoi usi e costumi - e in questo ricorda molto la connazionale Bibi della Michaelis. Una volta perso il padre in un incidente in mare, Pippi va a vivere da sola a Villa Villacolle, la casa che lui stava preparando per loro. Ha una cassa piena di monete d'oro, con le quali riesce a comprare tutto ciò di cui ha bisogno. Vive con una scimmietta e un cavallo. Non va a scuola: quando ci proverà non riuscirà a comprendere l'astrattezza dei metodi, quindi deciderà di non tornarci più. Ha una forza sovraumana, tanto da sollevare un cavallo - o due ladri, alloccorrenza -, "tuttavia essa usa i suoi poteri solo come difesa e la sua rivolta rimane circoscritta al mondo infantile: la società non la interessa". ${ }^{[12]}$ La bambina è talmente anticonvenzionale che i traduttori stranieri

[11] Cfr. ZILIOTTO, D., 2001, Pippi e non solo - Periodiche influenze della letteratura scandinava, in "Liber", n 51, p. 31.

[12] Cfr. ZILIOTTO, D., 1987, Generazione Bibi, generazione Pippi, in AA. VV., Bimbe donne e bambole - Protagoniste bambine nei libri per l'infanzia, Artemide edizioni, Roma, p. 30. 
apportano delle modifiche sostanziali alla storia, per renderla più credibile. La Lindgren ha il grande merito, sostiene a ragione Silvia Blezza Picherle, di "aver fatto uscire le protagoniste femminili dagli spazi interni (stanze di case e castelli) o circoscritti (giardini), dove le aveva da sempre confinate la letteratura per l'infanzia".[13] Pippi e le altre bambine dei suoi romanzi giocano infatti all'aperto e si arrampicano sugli alberi.

Anche Matilde, del romanzo omonimo di Roald Dahl (1989), è dotata di poteri magici che le permettono di sopravvivere agli adulti, acquisiti grazie alla sua portentosa intelligenza e utilizzati solo per far fronte alle ingiustizie a cui assiste. È in particolare grazie ai libri ed alla lettura che la bambina trova la forza per ribellarsi ai grandi, che sono prepotenti e crudeli con lei e con i suoi coetanei. Donatella Ziliotto, per la rivista "Liber", sostiene che Dahl abbia portato nella letteratura, anche italiana, una svolta senza precedenti. I bambini di Dahl sono armati contro la prepotenza e la crudeltà degli adulti e "le ostilità sono aperte seppur filtrate attraverso il grottesco e il surreale". ${ }^{[14]}$ I suoi personaggi, inoltre, sono supportati da un elemento magico utile a dare loro un sostegno: è un modo per sopperire alla mancanza di forza e potere che invece sono propri dei grandi.

Valentina (Le fatiche di Valentina, 1995) di Angelo Petrosino (1949) può essere annoverata tra i pochi esempi di bambine protagoniste di numerosi romanzi nella letteratura italiana (il suo autore ha dedicato a lei ed alle sue avventure più di centoquaranta romanzi). Questa non ha poteri magici, né è ribelle nei confronti degli adulti, ma è indipendente ed un punto di riferimento anche per i grandi che la circondano.

Bianca Pitzorno si inserisce perfettamente in questo contesto, in quanto prende a piene mani dalle esperienze maturate in prima persona, come lettrice appassionata di Carroll, della Michaelis e della Lindgren, per poi confrontarsi direttamente con Dahl (i cui romanzi più famosi sono stati pubblicati negli anni Ottanta, in tempi in cui sono anche pubblicati molti dei titoli dell'autrice stessa) e ancora di più con Petrosino, in quanto autori coetanei e connazionali.

[13] Cfr. BLEZZA PICHERLE, S., Un'inedita e "rivoluzionaria" rappresentazione dei personaggi, in BLEZZA PICHERLE, S., (a cura di), Rileggendo Astrid Lindgren - Percorsi critici e itinerari interpretativi, 2008, Edizioni Del Cerro, Pisa, p. 63.

[14] Cfr. ZILIOTTO, D., 2001, Pippi e non solo - Periodiche influenze della letteratura scandinava, in "Liber", n 51, p. 31 . 


\section{La lotta tra i sessi: confronti a distanza}

Le figure appena descritte possono essere considerate, a ragione, esempi luminosi di bambine ribelli e indipendenti della letteratura per l'infanzia, che non si accontentano dei ruoli che la società ha riservato loro, ma crescono inseguendo i propri sogni e aspirazioni. Tuttavia, come indicato in precedenza, gli autori assegnano raramente queste caratteristiche ai propri personaggi femminili. Emy Beseghi sostiene, infatti, che ci sia voluto del tempo prima che le bambine avessero il loro giusto spazio all'interno della letteratura per l'infanzia:

I romanzi per l'infanzia hanno da sempre offerto a maschi e femmine fantasie diverse spesso contrapposte, hanno suggerito sogni che sembrano prefigurare destini e modi di esistere. Schiacciate e devisualizzate nel racconto d'avventura - tradizionalmente "impero dei maschietti" -, confinate negli interni o cooptate precocemente nello spazio della donna adulta, le bambine sono state segno di una differenza elusa. Perché si affermi un cambiamento bisogna aspettare l'entrata in scena di donne che, attraverso la scrittura, hanno rivisitato il conflitto con il mondo adulto, con la propria crescita, con la propria appartenenza di genere. ${ }^{[15]}$

In particolare analizzando i cosiddetti "classici", ovvero quei libri che - secondo gli adulti - non possono mancare nella libreria di un bambino o di un adolescente, si può notare che ben pochi di essi hanno personaggi principali di sesso femminile, e quando li hanno sono relegati in un angolo - spesso non parlano neppure. La stessa Pitzorno in Bimbe donne e bambole ci parla di questa situazione:

Ma torniamo ai classici che furono per me fonte di incomparabili godimenti ed anche modelli letterari. Se richiamo alla mente i loro protagonisti sia pure in modo disorganico e confuso mi vedo circondata da una folla di maschi: bambini, ragazzi, uomini di tutte le età, nazionalità, caratteristiche fisiche, condizioni sociali... Vogliamo provare a pescare a caso nel mucchio? Robinson e il Piccolo Principe, Sandokan e Lord Fauntleroy, l'ultimo dei Moicani e Giamburrasca, Mowgli e il Capitano Nemo, Remy e David Copperfield, Pinocchio e Enrico Bottini, il Barone di Münchhausen e Nils Holgersson, Jim e Peter Pan, il Piccolo Alpino e Kim,

[15] Cfr. BESEGHI, E., Streghetta, Lavinia, Clorofilla e le altre in AA. VV., L'insegnante il testo l'allieva, 1992, Rosemberg e Sellier, Torino, p. 72. 
Gulliver, Christopher Robin, Tom Sawyer... C'erano anche libri, ricordo, che avevano per protagonisti una coppia mista di bambini o ragazzi. La bambina però era di solito una comprimaria un po' sacrificata, relegata nel 99\% dei casi in un ruolo "realistico", cioè subalterno e collaborativo, quando non era addirittura la piagnona che con la sua fragilità e le sue fisime rallentava il ritmo della avventura. ${ }^{[16]}$

Bianca Pitzorno, forse per un senso di rivalsa nei confronti di questa esclusione, per tutta la vita scrive quindi "storie di bambine e adolescenti autonome, coraggiose, non più spettatrici dell'azione", ${ }^{[17]}$ in lotta contro tutti quegli autori che, nel corso del tempo, le hanno ingiustamente relegate in un angolo dell'azione.

Questa caratteristica è lampante se si mettono a confronto alcuni dei Classici - citati sopra dalla stessa Pitzorno - con alcuni dei romanzi di maggior successo dell'autrice. Compiendo questa operazione è evidente che non solo le bambine di Bianca non hanno niente da invidiare ai "colleghi" maschi, ma che anzi sono migliori di loro sotto molti aspetti.

Accostando ad esempio Il barone rampante (1957) di Italo Calvino (1923-1985) a La casa sull'albero della Pitzorno (1984), ci troviamo di fronte a due protagonisti che condividono la scelta di andare a vivere sugli alberi - gioco riservato tradizionalmente ai soli bambini maschi. Nella lotta tra Cosimo e Aglaia è sicuramente la bambina che ha la meglio: la vita sugli alberi per lei è occasione di crescita, ma è sempre circondata dalle persone che le vogliono bene. La sua scelta di vita è in ogni momento reversibile (anche se non preferibile), mentre Cosimo rimane quasi imprigionato dal suo desiderio di libertà sugli alberi, arrivando fino a morire senza mai più toccare terra. Il romanzo La casa sullalbero insegna alle bambine (e ai bambini) che si può aspirare alla propria libertà, senza doversi per questo privare di nulla.

I due romanzi Le avventure di Pinocchio (1881) di Carlo Collodi (1826-1890) e La bambola dell'alchimista (1988) hanno invece in comune la presenza di un fantoccio che prende vita. Pinocchio, al pari della bambola Petra, abbandona le sue vesti inanimate per lasciare il posto ad un bambino in carne ed ossa. Solo che il Pinocchio burattino, perché subentri il bambino

\footnotetext{
[16] Cfr. PITZORNO, B., in AA. VV. Bimbe donne e bambole - Protagoniste bambine nei libri per l'infanzia, 1987, Artemide edizioni, Roma, pp. 38-39.

[17] Cfr. MANTELLASSI, F., 2017, La decostruzione degli stereotipi - Educazione e parità di genere in un percorso tra i libri che mirano all'abbandono di modelli precostituiti, in "Liber", n 113, p. 42.
} 
vero, deve morire: egli viene sostituito per lasciare il posto ad un bambino più obbediente. Per Petra, invece, non è così: i due bambini protagonisti, i "ribelli" Teo e Lavinia, non cambiano totalmente la loro natura nel diventare adulti, non ne hanno bisogno. Petra è il mezzo che li accompagna nel loro processo di maturazione personale. Anche questo sembra essere un atto di ribellione da parte di Bianca Pitzorno, che scrive:

Pinocchio l'ho rivalutato dopo i vent'anni, ma allora mi sembrò un libro cupo, pauroso, pieno di immagini di morte. Quegli assassini nel buio del bosco, e la casetta alla cui finestra si affaccia la bambina dai capelli turchini che diceva: 'Sono tutti morti. Anch'io sono morta', e poi la sua lapide con la scritta ricattatoria, e il burattino impiccato alla quercia grande, e i conigli vestiti di nero che trasportano la bara, e la tremenda metamorfosi in ciuchino, e il ciuchino affogato, e poi mangiato dai pesci... Ma la morte che mi stringeva più il cuore era quella finale del burattino. Perché al proposito non avevo dubbi. Lo scrittore voleva farci credere che Pinocchio si era trasformato in bambino, ma non era così. Uno che si trasforma, muta appunto la forma, ma conserva la sostanza di cui è fatto il corpo. Nel libro invece di corpi ce nerano due. Uno vivo e vispo: quello del ragazzino perbene; l'altro, quello del burattino, abbandonato sulla sedia, inerte, morto. Pinocchio era morto per lasciare il posto a un altro. Gli adulti, come Geppetto, non ci volevano bene così com'eravamo. Volevano che morissimo, per potersi prendere, al nostro posto, un altro bambino più bravo e obbediente. Era una storia vera e terribile: un episodio della guerra tra i grandi e i bambini, dove i bambini, pur dopo avere resistito valorosamente, venivano sconfitti e annientati. ${ }^{[18]}$

La bambola dell'alchimista, inoltre, può essere considerato un romanzo di vitale importanza non solo per le lettrici femmine, ma anche per i maschi: uno dei protagonisti della storia è infatti Teo, un bambino che, a discapito di tutte le convenzioni, gioca con le bambole.

Uno dei romanzi di maggior successo della Pitzorno è Ascolta il mio cuore (1991), volume che nasce come protesta nei confronti di Cuore (1886) di Edmondo de Amicis (1846-1908), romanzo contestato dall'autrice:

Non riusciva a convincermi. [...] Enrico Bottini, chera un bambino vero, aveva bisogno che gli dicesse tutto suo padre. Non era capace di commuoversi spontaneamente, di ammirare qualcosa, di fare un paragone, di vergognarsi, se non riceveva istruzioni dall'alto. Si era mai visto un

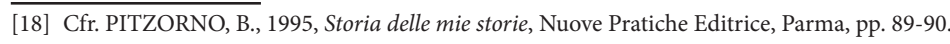


bambino così amorfo? Dovevano dirgli persino per chi doveva provare amicizia. Se lo avessero detto a me sarei diventata una belva. E poi tutte quelle lettere, fra gente che abitava nella stessa casa. Ed erano sempre i grandi a scrivergli. Lui non rispondeva mai. Fra l'altro il libro era scritto in prima persona, come se fosse lui a raccontare. Ma su queste lettere non si pronunciava mai. Possibile che fosse sempre d'accordo? Non aveva mai voglia di dire una parolaccia? Di scappare di casa? ${ }^{[19]}$

Entrambi i romanzi raccontano di un intero anno scolastico, vissuto nel caso di Ascolta il mio cuore da una bambina, e in quello di Cuore da un bambino, con esiti molto diversi. Quest'ultimo, pur facendo ancora presa nel cuore dei lettori, risente del tempo che passa: leggendolo oggi si ha l'impressione che tutto ciò che vi viene raccontato sia molto artificioso. Inoltre le (non) reazioni di Enrico inducono a pensare che questo bambino sia solo succube del mondo degli adulti, che pensano e agiscono per lui. Ascolta il mio cuore, invece, pur essendo anchesso ambientato nel passato, trova una risposta potente nell'animo delle bambine che oggi lo leggono. La distanza temporale non è di ostacolo, anzi: diventa un modo per riflettere sulle emozioni presenti e rispecchiarvisi con la giusta distanza. I sentimenti delle protagoniste sono reali, autentici, sempre veri: per questo risulta più attuale e godibile.

Bianca Pitzorno durante la sua carriera ha scritto diversi romanzi storici, rivolti a ragazzi e adulti, sfruttando le conoscenze acquisite durante gli anni di studi classici e archeologici. Uno dei più conosciuti è sicuramente Lamazzone di Alessandro Magno (1977), la cui protagonista Mìrtale è stata cresciuta dai lupi e poi data in dono al grande condottiero macedone. Questa origine ricorda immediatamente quella di Mowgli de Il libro della giungla (1893) di Rudyard Kipling (1865-1936), bambino-lupo che poi deve fare anch'egli ritorno tra gli uomini. Mìrtale è autonoma, autosufficiente in tutto e fin da subito. Pur condividendo l'origine di Mowgli, essa ha sì come lui dei mentori, ma è sempre in grado di prendere le proprie decisioni indipendentemente da loro. Quando si tratta di scegliere della propria vita, Mìrtale compie un gesto davvero coraggioso: tornare nella giungla, rinunciando agli agi di una vita seguendo la carovana di Alessandro. È lei che, a dispetto di tutto e di tutti, sola, prende questa decisione. Mowgli [19] Ivi, pp. 90-91. 
decide invece di tornare tra gli uomini perché sa che è la cosa giusta da fare, applicando però la Legge della Giungla. La storia di Mìrtale ricorda anche quella di un altro romanzo di Kipling, Kim (1901). Mìrtale decide, al pari del protagonista di quest'ultimo libro, di diventare una discepola di un santone indiano, seguendo la via della beatitudine, del nirvana. Scrive a questo proposito Mirca Casella:

Anche Mìrtale, come Kim, viene istruita, ed entrambi hanno la possibilità di seguire la via del popolo più forte. La bambina della Pitzorno però vuole cercare da sola la sua strada, abbandona Alessandro e tutto quello che lui rappresenta per seguire se stessa e il vecchio saggio, come Kim è aiutato a diventare adulto dall'esperienza di vita compiuta con il maestro. Kim non sceglie drasticamente; entra a far parte del servizio segreto britannico ma nello stesso tempo gioisce per aver acquistato la Salvezza insieme al lama. Mìrtale compie la sua scelta e lascia la carovana che rappresenta la sua casa e la sua vita: di lei nessuno ci ha raccontato più nulla, ma è lecito, per il lettore, continuare a pensare, come succede per Kimball O’Hara, al seguito della sua storia. ${ }^{[20]}$

Mowgli, Kim e Mìrtale hanno tutti e tre un destino comune: cercare le proprie origini. Solo in questo modo possono abbracciare le loro vite e continuare a crescere, per diventare adulti. La differenza più grande si trova nel fatto che le decisioni di Mowgli e di Kim siano molto guidate dagli altri personaggi che ruotano intorno a loro, mentre Mìrtale compie le sue scelte in totale autonomia.

Infine può essere analizzato un ultimo volume, Polissena del porcello (1993), che si propone come parodia e insieme omaggio al feuilleton ottocentesco, genere amatissimo da Bianca Pitzorno e di cui Senza famiglia (1878) di Hector Malot (1830-1907) è uno degli elementi di spicco. I punti che accomunano i due romanzi sono molto numerosi: entrambi i protagonisti scoprono di non essere figli di quelli che credevano essere i propri genitori e partono alla ricerca della loro vera famiglia; entrambi incontrano i veri genitori senza riconoscerli e invece ne identificano altri che alla fine si rivelano essere impostori; nei romanzi compare l'elemento dei saltimbanco e degli animali ammaestrati; un bambino povero scopre

[20] Cfr. CASELLA, M., 2006, Le voci segrete - Itinerari di iniziazione al femminile nellopera di Bianca Pitzorno, Mondadori, Milano, pp. 108-109. 
di avere sangue aristocratico; infine per l'avventura partono due amici, di cui uno molto povero, che per vivere fanno spettacoli per la strada. Nella storia di Rémi troviamo i sentimenti e le emozioni camuffati - pur essendo il romanzo raccontato in prima persona, come fosse un libro di memorie. Rémi è sempre forte, coraggioso, generoso, allegro, non si perde mai d'animo. È un "bambino di carta" in tutto e per tutto, ma sulla carta sembra rimanere, perché non è verosimile. Polissena, invece, è un personaggio che si stacca dalla pagina del libro e prende vita: è una bambina che, pur vivendo storie assurde e molto distanti dalla realtà, ci sembra di poter conoscere appieno.

\section{Conclusioni}

Bianca Pitzorno, da piccola, da una parte si sente molto fiera del suo essere una femmina - specialmente grazie ad una nonna che la preferisce a tutti gli altri nipoti proprio per questo motivo - ma dall'altra è una "bambina molto arrabbiata", ${ }^{[21]}$ perché si rende conto che sono sempre i maschi della famiglia ad avere le maggiori libertà e le migliori possibilità.

Da sempre è una lettrice appassionata, ma purtroppo la letteratura per l'infanzia non sta dalla parte delle bambine. I personaggi femminili, come abbiamo visto, sono il più delle volte portati in secondo piano o messi da parte - quando essi sono presenti. La maggior parte dei protagonisti che incarnano qualità positive e che rappresentano un esempio per i giovani lettori sono di sesso maschile. Non è un caso, tuttavia, che l'autrice si appassioni in particolare al ciclo di Sandokan: spesso i romanzi di Emilio Salgari parlano infatti di donne non solo belle, ma anche forti, coraggiose e intelligenti.

Emy Beseghi conia una magnifica espressione per definire la Pitzorno: "madre letteraria di sole figlie femmine". ${ }^{[22]}$ Bianca Pitzorno va a colmare così un importante vuoto nella letteratura per l'infanzia: quello lasciato dagli scrittori che, a parte qualche splendida eccezione già presentata nel primo paragrafo, hanno da sempre preferito i maschi alle femmine.

[21] PITZORNO, B., 1995, Storia delle mie storie, Nuove Pratiche Editrice, Parma.

[22] BESEGHI, E., Polissena nel labirinto di Gaia, in BESEGHI, E., (a cura di), Nel giardino di Gaia, 1994, Mondadori, Milano, p. 70. 


\section{Bibliografia}

AA. VV., Bimbe donne e bambole - Protagoniste bambine nei libri per l'infanzia, 1987, Artemide edizioni, Roma.

AA. VV., L'insegnante il testo l'allieva, 1992, Rosemberg e Sellier, Torino.

AA. VV., "Liber - Libri per bambini e per ragazzi", Edificanti o sovversivi, $\mathrm{n}^{\circ}$ 51, 2001 (rivista).

AA. VV., "Liber - Libri per bambini e per ragazzi", Le storie degli altri, $\mathrm{n}^{\circ} 113,2017$ (rivista).

BLEZZA PICHERLE, S., (a cura di), Rileggendo Astrid Lindgren Percorsi critici e itinerari interpretativi, 2008, Edizioni Del Cerro, Pisa.

BLEZZA PICHERLE, S., 2004, Libri, bambini, ragazzi - Incontri tra educazione e letteratura, Vita e Pensiero, Milano.

CALVINO, I., 1991, Il barone rampante, in Romanzi e racconti, Mondadori, Milano.

CARROLL, L., 2010, Alice nel Paese delle Meraviglie, Grandi Tascabili Economici Newton, Roma.

CASELLA, M., 2006, Le voci segrete - Itinerari di iniziazione al femminile nell'opera di Bianca Pitzorno, Mondadori, Milano.

CATARSI, E., (a cura di), 2002, Gianni Rodari e la letteratura per l'infanzia, Edizioni Del Cerro, Pisa.

COLLODI, C., 2001, Pinocchio, Piemme, Casale Monferrato.

DAHL, R., 1982, Matilde, Salani, Milano.

DE AMICIS, E., 1991, Cuore, De Agostini Ragazzi, Milano.

KIPLING, R., 1998, Il libro della Giungla, Einaudi Editore, Torino.

KIPLING, R., 2013, Kim, Rizzoli Editore, Milano.

LINDGREN, A., 2012, Pippi Calzelunghe, RCS Periodici, Milano.

MALOT, H., 2001, Senza famiglia, Fabbri Editori, Milano.

MICHAELIS, K., 1931, Bibi - Una bimba del nord, Villardi, Milano.

PETROSINO, A., 1995, Le fatiche di Valentina, Piemme, Casale Monferrato.

PITZORNO, B., 1995, Storia delle mie storie, Nuove Pratiche Editrice, Parma.

PITZORNO, B., 1991, Ascolta il mio cuore, Mondadori, Milano. 
PITZORNO, B., 1977, L’amazzone di Alessandro Magno, Rusconi, Milano.

PITZORNO, B., 1988, La bambola dell'alchimista, Mondadori, Milano.

PITZORNO, B., 1984, La casa sull'albero, Le Stelle, Milano.

PITZORNO, B., 1993, Polissena del Porcello, Mondadori, Milano.

\section{Djevojčice - na njihovoj strani}

Analiza doprinosa dječjoj književnosti Biance Pitzorno

Predmet rada je panoramski prikaz prinosa dječjoj književnosti talijanske spisateljice Biance Pitzorno. Autorica je važna na nacionalnoj i međunarodnoj razini jer je svoj život posvetila pisanju priča o djevojčicama i adolescenticama koje je književnost udaljila u kut, povjeravajući im sekundarne uloge ili slabe i podložne likove. U literaturi nalazimo i druge autore koji su pisali o djevojčicama (kao što su Carroll, Michaelis, Lindgren, Dahl i Petrosino), ali im se nitko nije posvećivao u potpunosti kao Pitzorno. Uspoređujući neke muške protagoniste klasika (osobito Calvina, Collodija, De Amicisa, Kiplinga i Malota) sa ženskim likovima Biance Pitzorno pokazalo se da one nemaju što zavidjeti "muškim kolegama".

Ključne riječi: Pitzorno, djevojčice, književnost, stereotipi, spol

\section{The Girls' Side \\ Analysis of Bianca Pitzorno's Contribution to Children's Literature}

This essay provides an overview of the contribution of the Italian writer Bianca Pitzorno to children's literature. This author is important on both a national and international level. She dedicated her life to writing stories with female children and teenagers as lead characters when the literature of the mostly provided them with supporting roles with weak or submissive personalities. Other literary authors wrote about girls (e.g. Carroll, Michaelis, Lindgren, Dahl and Petrosino), but none of them ever embraced this task like Pitzorno. Also if we compare some of lead male 
characters in classic children's literature (ones by Calvino, Collodi, De Amicis, Kipling and Malot) with Pitzorno's female ones, we can see that the latter are on par with their male counterparts.

Key words: Pitzorno, girls, literature, stereotypes, gender 